\title{
DEDEKIND SUMS AND LAMBERT SERIES
}

L. CARLITZ

1. Introduction. Apostol [1] has proved a transformation formula for the function

$$
G_{p}(x)=\sum_{m, n=1}^{\infty} n^{-p} x^{m n}
$$

where $p$ is a fixed odd integer $>1$. In the formula occur the numbers

$$
c_{s}(h, k)=\sum_{\mu(\bmod k)} \bar{B}_{p+1-8}\left(\frac{\mu}{k}\right) \bar{B}_{s}\left(\frac{h \mu}{k}\right) \quad(0 \leqq s \leqq p+1),
$$

where $(h, k)=1$, the summation is over a complete residue system $(\bmod k)$, and $\bar{B}_{s}(x)$ is the Bernoulli function. Put

$$
f(h, k ; \tau)=\sum_{s=0}^{p+1}\left(\begin{array}{c}
p+1 \\
s
\end{array}\right)(k \tau-h)^{p-s} c_{s}(h, k) ;
$$

then Apostol's formula can be put in the form $[2, \S 2]$

$$
G_{p}\left(e^{2 \pi i \tau}\right)=(k \tau-h)^{p-1} G_{p}\left(e^{2 \pi i \tau^{\prime}}\right)+\frac{(2 \pi i)^{p}}{2(p+1) !} f(h, k ; \tau),
$$

where $\tau=\left(h^{\prime} \tau+k^{\prime}\right) /(k \tau-h), h h^{\prime}+k k^{\prime}+1=0$. It is also shown in [2] that (1.3) implies the following transformation formula for $f(h, k ; \tau)$ :

$$
f(h, k ; \tau)=\tau^{p-1} f\left(-k, h ;-\frac{1}{\tau}\right)+\frac{1}{\tau}(B+\tau B)^{p+1} .
$$

The purpose of this note is to give an elementary proof of (1.4) depending on the representation of $c_{s}(h, k)$ by means of "Eulerian" numbers (see (2.3) below).

2. It is convenient to use, in place of (1.1), the fuller notation

$$
c_{r, 8}(h, k)=\sum_{\mu(\bmod k)} \bar{B}_{r}\left(\frac{\mu}{k}\right) \bar{B}_{s}\left(\frac{h \mu}{k}\right),
$$

where now $r, s$ are arbitrary non-negative integers; thus it is clear that $c_{s}(h, k)=c_{p+1-8, s}(h, k)$. If we define the Eulerian numbers $H_{m}(\alpha)$ by means of

Presented to the Society, February 27, 1954; received by the editors January 19, 1954. 


$$
\frac{1-\alpha}{e^{x}-\alpha}=\sum_{m=0}^{\infty} H_{m}(\alpha) \frac{x^{m}}{m !}
$$

then we have the representation $[2, \S 6]$

$$
c_{r, s}(h, k)=\frac{B_{r} B_{s}}{k^{r+s-1}}+\frac{r s}{k^{r+s-1}} \sum_{\zeta \neq 1} \frac{H_{r-1}\left(\zeta^{h}\right)}{1-\zeta^{-h}} \frac{H_{s-1}\left(\zeta^{-1}\right)}{1-\zeta},
$$

where $B_{m}$ denotes a Bernoulli number in the even suffix notation and the summation is over all $k$ th roots of unity distinct from 1 . The formula (2.3) is proved for $r \geqq 1, s \geqq 1$ but if we interpret the sum in the right member as 0 for $r s=0$, then it is easily verified that (2.3) is valid for all non-negative $r, s$. In the next place we have, using (2.2),

$$
\begin{aligned}
\sum_{r, s=0}^{\infty} k^{r} c_{r, s}(h, k) & \frac{x^{r} y^{s}}{r ! s !}=\frac{x}{e^{x}-1} \frac{y}{e^{y / k}-1} \\
& +x y \sum_{\zeta \neq 1} \frac{1}{\left(1-\zeta^{-h}\right)(1-\zeta)} \sum_{r=0}^{\infty} H_{r}\left(\zeta^{h}\right) \frac{x^{r}}{r !} \sum_{s=0}^{\infty} H_{s}\left(\zeta^{-1}\right) \frac{y^{s} k^{-s}}{s !} \\
= & \frac{x}{e^{x}-1} \frac{y}{e^{y / k}-1}+x y \sum_{\zeta \neq 1} \frac{\zeta^{h}}{e^{x}-\zeta^{h}} \frac{\zeta^{-1}}{e^{y / k}-\zeta^{-1}}
\end{aligned}
$$

and therefore

$$
\sum_{r, s=0}^{\infty} k^{r} c_{r, s}(h, k) \frac{x^{r}}{r !} \frac{y^{s}}{s !}=x y \sum_{\zeta} \frac{\zeta^{h}}{e^{x}-\zeta^{h}} \frac{\zeta^{-1}}{e^{y / k}-\zeta^{-1}},
$$

where the summation is now over all $k$ th roots of unity.

If we put

$$
b_{r, s}(h, k)=\sum_{t=0}^{s}(-1)^{s-t}\left(\begin{array}{l}
s \\
t
\end{array}\right) h^{s-t} c_{r+s-t, t}(h, k)
$$

or, what is the same thing,

$$
c_{r, s}(h, k)=\sum_{t=0}^{s}\left(\begin{array}{l}
s \\
t
\end{array}\right) h^{s-t} b_{r+s-t, t}(h, k),
$$

then a straight-forward computation yields

$$
\sum_{r, s=0}^{\infty} k^{r} c_{r, s}(h, k) \frac{x^{r}}{r !} \frac{y^{s}}{s !}=\sum_{r, s=0}^{\infty} b_{r, s}(h, k) \frac{(k x+h y)^{r}}{r !} \frac{y^{s}}{s !} .
$$

For brevity we put

$$
k z=k x+h y
$$


and

$$
F(h, k ; z, y)=\sum_{r, s=0}^{\infty} b_{r, s}(h, k) \frac{z^{r}}{r !} \frac{y^{s}}{s !} .
$$

It follows from (2.4) and (2.7) that

$$
F(h, k ; k z, y)=x y \sum_{\zeta} \frac{\zeta^{h}}{e^{x}-\zeta^{h}} \frac{1}{e^{y / k} \zeta-1} .
$$

3. If $\eta$ runs through the $h$ th roots of unity, then

$$
\frac{h}{x^{h}-1}=\sum_{\eta} \frac{\eta}{x-\eta}=\sum_{\eta} \frac{1}{x \eta-1} .
$$

Thus

$$
\frac{h \zeta^{h}}{e^{x}-\zeta^{h}}=\frac{h}{e^{x} \zeta^{-h}-1}=\sum_{\eta} \frac{1}{e^{x / h} \eta \zeta^{-1}-1}=\sum_{\eta} \frac{\zeta}{e^{x / h} \eta-\zeta}
$$

and (2.10) becomes

$$
F(h, k ; k z, y)=\frac{x y}{h} \sum_{\eta, \zeta} \frac{\zeta}{e^{x / h} \eta-\zeta} \frac{1}{e^{y / k} \zeta-1} .
$$

But since

$$
\frac{\zeta}{e^{x / h} \eta-\zeta} \frac{1}{e^{y / k} \zeta-1}=\left(\frac{\eta e^{x / h}}{e^{x / h} \eta-\zeta}+\frac{1}{e^{y / k} \zeta-1}\right) \frac{1}{e^{x / h+y / k} \eta-1}
$$

the right member of (3.2) is equal to

$$
\frac{x y}{h} \sum_{\eta, \zeta} \frac{\eta e^{x / h}}{e^{x / h} \eta-\zeta} \frac{1}{e^{x / h+y / k} \eta-1}+\frac{x y}{h} \sum_{\eta, 5} \frac{1}{e^{y / k} \zeta-1} \frac{1}{e^{x / h+y / k} \eta-1} .
$$

Then using (2.8) and (3.1) we get

$$
\begin{aligned}
F(h, k ; k z, y)= & -\frac{k x y}{h} \sum_{\eta} \frac{1}{e^{-k x / h} \eta^{-k}}-1 \frac{1}{e^{z / h} \eta-1} \\
& +\frac{k x y}{\left(e^{y}-1\right)\left(e^{z}-1\right)} .
\end{aligned}
$$

Returning to (2.14), we replace $h, k, x, y$ by $k, h,-k x / h, z$ respectively; we get

$$
\text { (3.4) } \sum_{r, s=0}^{\infty} c_{r, s}(k, h) \frac{(-k x)^{r}}{r !} \frac{z^{s}}{s !}=-\frac{k x z}{h} \sum_{\eta} \frac{1}{e^{-k x / h} \eta^{-k}-1} \frac{1}{e^{z / h} \eta-1} \text {. }
$$


But by (2.7) the left-hand member of (3.4) is equal to

$$
\sum_{r, s=0}^{\infty} b_{r, s}(k, h) \frac{(k z-k x)^{r}}{r !} \frac{z^{s}}{s !}=\sum_{r, s=0}^{\infty} b_{r, s}(k, h) \frac{(h y)^{r}}{r !} \frac{z^{s}}{s !} .
$$

Comparison with (3.3) leads at once to

(3.5) $z F(h, k ; k z, y)=y F(k, h ; h y, z)+(k z-h y) \frac{z}{e^{z}-1} \frac{y}{e^{y}-1} \cdot$

4. We now compare coefficients of $z^{r} y^{s}$ in both members of (3.5). In view of (2.9) we get at once

$$
r k^{r-1} b_{r-1,8}(h, k)=s h^{s-1} b_{s-1, r}(k, h)+r k B_{r-1} B_{s}-s h B_{r} B_{s-1}
$$

for all $r, s \geqq 0$. Incidentally this proves Theorem 1 of [2].

If we put

$$
f_{m}(h, k ; \tau)=\sum_{s=0}^{m}\left(\begin{array}{c}
m \\
s
\end{array}\right)(k \tau-h)^{m-1-8} c_{m-s, s}(h, k),
$$

then it follows readily from $(2.5)$ that

$$
(k \tau-h) f_{m}(h, k ; \tau)=\sum_{s=0}^{m}\left(\begin{array}{c}
m \\
s
\end{array}\right)(k \tau)^{m-s} b_{m-8,8}(h, k) .
$$

In (4.3) replace $h, k, \tau$ by $-k, h,-1 / \tau$, respectively; then

$$
\begin{aligned}
\frac{k \tau-h}{\tau} f_{m}(-k, h ;- & \left.\frac{1}{\tau}\right) \\
& =\sum_{s=0}^{m}\left(\begin{array}{c}
m \\
s
\end{array}\right)\left(-\frac{h}{\tau}\right)^{m-s} b_{m-s, s}(-k, h) .
\end{aligned}
$$

But it is clear from (2.1) that

$$
c_{r, s}(-k, h)=(-1)^{s} c_{r, s}(k, h)
$$

and therefore (2.5) implies

$$
b_{r, s}(-k, h)=(-1)^{s} b_{r, s}(k, h) .
$$

Hence (4.4) becomes

$$
\tau^{m-1}(k \tau-h) f_{m}\left(-k, h ;-\frac{1}{\tau}\right)
$$

$$
=(-1)^{m} \sum_{s=0}^{m}\left(\begin{array}{c}
m \\
s
\end{array}\right) \tau^{s} h^{m-s} b_{m-s, 8}(k, h) .
$$


It follows that

$$
\begin{aligned}
& (k \tau-h)\left\{f_{m}(h, k ; \tau)-(-1)^{m} \tau^{m-2} f_{m}\left(-k, h ;-\frac{1}{\tau}\right)\right\} \\
& =\sum_{s=0}^{m}\left(\begin{array}{c}
m \\
s
\end{array}\right) \tau^{m-s}\left\{k^{m-s} b_{m-s, s}(h, k)-\frac{s}{m-s+1} h^{s-1} b_{s-1, m-s+1}(k, h)\right\} \\
& =\sum_{s=0}^{m}\left(\begin{array}{c}
m \\
s
\end{array}\right) \tau^{m-s}\left\{k B_{m-s} B_{s}-\frac{s}{m-s+1} h B_{m-s+1} B_{s-1}\right\} \\
& =k \sum_{s=0}^{m}\left(\begin{array}{c}
m \\
s
\end{array}\right) B_{m-s} B_{s}-h \sum_{s=1}^{m+1}\left(\begin{array}{c}
m \\
s-1
\end{array}\right) \tau^{m-s} B_{m-s+1} B_{s-1} \\
& =\left(k-\frac{h}{\tau}\right)(B+\tau B)^{m},
\end{aligned}
$$

and therefore

(4.7) $f_{m}(h, k ; \tau)=(-1)^{m} \tau^{m-2} f_{m}\left(-k, h ;-\frac{1}{\tau}\right)+\frac{1}{\tau}(B+\tau B)^{m}$.

In particular for $m=p+1, p$ odd, (4.7) reduces to (1.4).

We have therefore proved (1.4) and indeed the more general result (4.7) using only the representation (2.3) and familiar properties of the Bernoulli functions.

\section{REFERENCES}

1. T. M. Apostol, Generalized Dedekind sums and transformation formulae of certain Lambert series, Duke Math. J. vol. 17 (1950) pp. 147-157.

2. L. Carlitz, Some theorems on generalized Dedekind sums, Pacific Journal of Mathematics vol. 3 (1953) pp. 513-522.

DURE UNIVERSITY 